\title{
A INTERAÇÃO ENTRE GRANDES EMPRESAS E STARTUPS: DESAFIOS DA INOVAÇÃO ABERTA
}

\author{
Janice Rodrigues Maciel ${ }^{1}$, Juliana Baldessar Weber Becker ${ }^{2}$, Rossana Alves de Oliveira \\ Simão Gomes ${ }^{3}$, Clarissa Stefani Teixeira ${ }^{4}$ \\ 1janicemaciel@gmail.com \\ 2jubweber@gmail.com \\ ${ }^{3}$ rossanaoliveira.alves@gmail.com \\ ${ }^{4}$ clastefani@gmail.com
}

\section{Resumo}

A inovação é hoje imperativo para a sobrevivência de qualquer negócio. Grandes empresas, que costumavam concentrar internamente seus esforços de inovação, pesquisa e desenvolvimento, passaram a adotar a inovação aberta, É neste contexto, que este artigo busca, por meio de uma revisão bibliográfica, explorar as interações que têm surgido entre grandes empresas e startups. Verifica-se como resultado dessa interação que as empresas se beneficiam com a agilidade na solução de problemas com menor custo e risco e as startups pelo maior acesso à infraestrutura, recursos financeiros e aumento de visibilidade do mercado. Por outro lado, há também desafios a serem enfrentados, tais como discrepância entre a cultura organizacional, proteção da propriedade intelectual e avaliação de ativos.

Palavras Chave: Inovação aberta. Startup. Interação.

\section{THE INTERACTION BETWEEN BIG COMPANIES AND STARTUPS - CHALLENGES OF OPEN INNOVATION}

\begin{abstract}
Innovation is mandatory today for the survival of any business. Large companies, that usually focused internally their innovation, research and development efforts, began to adopt open innovation. It is in this context, that this article seeks, through a bibliographic review, to explore the interactions that have arisen between large companies and startups. As a result of this interaction, companies benefit from agility in solving problems with lower cost and risk and startups by greater access to infrastructure, financial resources and increased market visibility. On the other hand, there are also challenges to be faced, such as discrepancy between organizational culture, intellectual property protection and asset valuation.
\end{abstract}

Keywords: Open innovation. Startup. Interaction.

\section{Introdução}

O termo inovação aberta foi inicialmente definido como o uso de entradas e saídas intencionais de conhecimento para acelerar a inovação interna e expandir os mercados pelo uso externo da inovação (Chesbrough, 2003). Sob o paradigma da inovação aberta, existe um fluxo importante de conhecimento externo à organização que se transforma em projetos de cooperação com parceiros externos, incentivando a compra e incorporação de tecnologias externas (Rahman e Ramos, 2010).

As formas para se conduzir a inovação aberta podem ser diversas. Tradicionalmente, a interação com universidades e centros de pesquisa e tecnologia se constituiu como a principal

R. Eletr. do Alto Vale do Itajaí - REAVI, v. 7, n. 11, p. 01-09, dez., 2018 ISSN: 2316-4190, DOI: $10.5965 / 2316419007112018001$ 
estratégia para aquisição de conhecimento externo (Stal. Nohara e Chagas, 2014). Atualmente, um dos caminhos explorados pelas grandes empresas é a interação com startups, uma tendência que ganha cada vez mais força. Varrichio (2016) aponta que as grandes empresas estão buscando nas startups, uma maneira de diversificar suas estratégias de inovação e acessar novas tecnologias a um custo de investimento relativamente baixo.

No Brasil, a relação entre empresas e startups tem se mostrado crescente, porém ainda muito aquém do nível internacional (ABDI, 2017). Este movimento vem se intensificando e, segundo pesquisa realizada pela 100 Open Startups (2017), num período de dois anos, entre 2015 e 2017, foram 613 contratos gerados entre startups e grandes empresas e mais de 150 programas formais de relacionamento entre as partes. Entretanto, dados referentes às práticas de inovação aberta entre grandes empresas e startups ainda são inexistentes, principalmente na literatura nacional.

Neste sentido, o objetivo geral do presente estudo é analisar as oportunidades e desafios da interação entre grandes empresas e startups, no contexto da inovação aberta.

\section{Referencial Teórico}

A rápida e constante evolução das tecnologias, novos modelos de negócios e novas formas de consumo vêm mudando a forma de se conduzir negócios. A inovação passa a figurar com um eixo central da estratégia das empresas que buscam sobreviver num ambiente de cada vez mais incerteza e imprevisibilidade. Para prosperar neste cenário inovar é um imperativo (Sudati, 2016).

Tradicionalmente, inovação pode ser considerada como um processo que abarca as atividades técnicas, concepção, desenvolvimento e gestão que tem como resultado a comercialização de novos ou melhorados produtos (OCDE, 2002). No que denominam inovação fechada, as empresas buscam soluções dentro do seu próprio corpo técnico, ou seja, investem fortemente em P\&D e se empenham para que todo o processo de inovação permaneça interno e assim as ideias são geradas, desenvolvidas, produzidas e lançadas no mercado pela própria empresa (Chesbrough, 2003). Por muito tempo essa filosofia foi tida como a mais correta, posto que entendiam que esse caminho conduzia a um ciclo virtuoso de inovação, onde se colhia altos lucros e estes eram reinvestidos em P\&D (Docherty, 2006). A alta competitividade do mercado e a celeridade com que as tecnologias se tornam obsoletas, fez com que a prática antes tida como ideal sofresse alteração de paradigma, levando às empresas a mudarem suas perspectivas (Chesbrough, 2003). O autor afirma que "o modelo tradicional de inovação - amplamente focado no ambiente interno, fechado de ideias e tecnologias externas está ficando obsoleto". Além disso, a massificação das tecnologias da informação e comunicação levou ao aumento da mobilidade de profissionais entre empresas concorrentes e entre indústrias, fazendo com que o conhecimento se difundisse mais facilmente entre as empresas, o que acelerou a passagem do modelo típico de "inovação fechada" para o modelo de "inovação aberta" (Nirazawa, 2015).

Nesse sentido, surge como alternativa um modelo diferenciado de inovar, a inovação aberta (Engeroff e Balestrin, 2008). Contudo, não se trata necessariamente de extinguir os departamentos internos de $\mathrm{P} \& \mathrm{D}$, posto que esses permanecem úteis para definição do que se busca fora, bem como aplicação dos resultados obtidos (Stal. Nohara e Chagas, 2014).

Cunhado por Chesbrough, o termo inovação aberta foi definido como o uso intencional de fluxos de entrada e de saída de conhecimentos com o propósito de respectivamente acelerar a inovação interna e expandir os mercados para o uso externo de inovação (Chesbrough, Vanhaverbeke e West, 2006). Trata-se de uma estratégia para acessar conhecimento externo, tornando o processo de inovação mais rápido, mais dinâmico e mais barato.

R. Eletr. do Alto Vale do Itajaí - REAVI, v. 7, n. 11, p. 01-09, dez., 2018 ISSN: 2316-4190, DOI: $10.5965 / 2316419007112018001$ 
Os princípios que caracterizam inovação aberta são, para Chesbrough (2006): cooperar estreitamente com os clientes no desenvolvimento de novas soluções; focar na utilidade, não no produto; explorar os talentos externos à empresa; a $\mathrm{P} \& \mathrm{D}$ externa pode trazer muito valor para a empresa, enquanto a $P \& D$ interna é fundamental para a apropriação de parte desse valor; e não é preciso estar na origem da investigação para tirar proveito dela.

Chesbrough (2003) cita ainda alguns dos fatores determinantes para a consolidação desta tendência: a globalização, que permitiu que as empresas passassem a ter acesso a conhecimento e recursos de todo o mundo, facilitado por tecnologias da informação que tornaram possível a descentralização e a virtualização dos processos de pesquisa e desenvolvimento; a terceirização que intensificou a especialização e tornou mais fragmentada as cadeias de valor industriais; a integração da visão do cliente e fornecedores, contribuindo no processo integrado de inovação e desenvolvimento de produtos e a reorientação a novos modelos de negócio para além do mercado tradicional.

Huizingh (2011), no entanto, pontua que estas práticas não são novidades, mas no conjunto forçaram as empresas a reconsiderarem seus processos gerenciais internos e se tornarem mais abertas, passando do modelo fechado de inovação para um modelo aberto e colaborativo.

Nisto, surgem diversos tipos e métodos de se inovar de forma aberta (Lazzorotti, Manzini, 2009), que podem variar no tipo de relação que a empresa estabelece com seus parceiros; no tipo de parceiro (Chiesa; Manzini; Pizzurno 2004), na forma de aquisição ou exploração do conhecimento externo e na etapa do processo inovador (Berger Et Al., 2005).

São diversas também as fontes de colaboração e obtenção de conhecimento para a inovação, que podem vir de universidades, institutos de pesquisa e desenvolvimento, cadeia de fornecedores, clientes, crowdsourcing, concorrentes, etc . E é neste contexto que surgiu, na última década, uma interação particular entre grande empresa e startups (Weiblen, Chesbrough, 2015; Sudati 2016). Embora bastante distintas entre si, estes dois tipos de corporações possuem características que se complementam (Weiblen, Chesbrough, 2015). Faltam às grandes empresas, a adaptabilidade que as startups possuem. Em contraponto, faltam às startups o poder econômico e de mercado detidos pelas grandes empresas.

Desta assimetria, dois pontos são relevantes na discussão: os desafios decorrentes da interação entre as partes e as diversas oportunidades e geração de negócios que surgem.

\section{Procedimentos Metodológicos}

O presente estudo pode ser caracterizado como exploratório, pois teve como objetivo o aprimoramento de ideias e familiarização das pesquisadoras em relação aos fatos relacionados ao tema escolhido e ao problema da pesquisa (Santos, 2006). Um levantamento bibliográfico foi realizado com intuito de caracterização do conteúdo necessário para apropriação do tema e criação de base teórica para discussão (Gil, 2002).

As análises foram extraídas de artigos científicos, teses, dissertações, sítios institucionais e livros . Os dados foram obtidos por meio de pesquisa em base de dados como Scielo e Scopus, websites nacionais e estrangeiros, com variações dos seguintes termos: Inovação Aberta; Empresas; Startups; Desafios / Open Innovation; companies; Startups; Challenges. Ademais foram utilizadas obras referenciadas em artigos e teses e também outros textos recomendados por especialistas da área.

Por meio desta base teórica obteve-se embasamento suficiente para abordar o problema da pesquisa.

\section{Discussão}

R. Eletr. do Alto Vale do Itajaí - REAVI, v. 7, n. 11, p. 01-09, dez., 2018 ISSN: 2316-4190, DOI: $10.5965 / 2316419007112018001$ 
A interação entre grande empresa e startups como uma estratégia de inovação aberta intensificou-se de modo expressivo na última década, vista como uma possível prática gerencial para institucionalizar o empreendedorismo e consequentemente, implementar uma estratégia de inovação contínua (Sudati, 2016).

Embora sejam bastante distintas em suas características, a parceria pode beneficiar de modo expressivo ambas as partes (Weiblen, Chesbrough, 2015). A agilidade, velocidade e o conhecimento tecnológico das startups chamam a atenção das grandes empresas, enquanto os recursos, canais comerciais, networking, capacidade produtiva e a estrutura financeira destas últimas, podem contribuir para o sucesso das startups (Hogenhuis; Hende; Hultink, 2016).

Segundo Thieme (2017), as grandes empresas são caracterizadas pela lentidão nas decisões, dificuldade para inovar e baixa exposição ao risco (Thieme, 2017). Devido ao tamanho, seus processos se tornam mais burocráticos e menos flexíveis, levando a uma morosidade que, na geração de inovação, é altamente maléfico (Weiblen, Chesbrough, 2015).

Startups são empresas, em geral de base tecnológica, que operam em um cenário de grande risco e incertezas, e orientadas para um rápido crescimento (Graham, 2012). Costumam se posicionar na fronteira da inovação, são intensivas em conhecimento especializado e mais flexíveis para assumir riscos, explorar novas tecnologias e novos modelos de negócio (Hogenhuis; Hende; Hultink, 2016).

Thieme (2017) defende que grandes empresas estão voltadas à execução de um modelo de negócios escalável, enquanto as startups exploram novos modelos de negócios escaláveis. Por consequência desta orientação, surge a necessidade da interação com startups, como forma da grande empresa sair da inércia e inovar.

Genericamente denominada Corporate-Startup Engagement (CSE) (Weiblen, Chesbrough, 2015), a interação entre grandes empresas e startups possuem dois fatores importantes a serem definidos: os objetivos da interação e, a partir disto, as formas como esta interação se materializará. Esta visão é compartilhada por Hogenhuis, Hende e Hultink (2016), para os quais, em que pesem todos os benefícios e oportunidades decorrentes destas interações, é fundamental se estabelecer um modelo claro de tomada de decisões para a escolha do parceiro correto, com o intuito de minimizar as assimetrias entre as partes, chamando a atenção para a escolha do estágio ideal do projeto no qual desejam colaboração e as capacidades do parceiro, definindo como ambas partes podem se beneficiar, em uma transação ganha-ganha (Vermeersch, Vanhaverbeke, Zutter, 2012; Hogenhuis, Hende, Hultink, 2016).

De acordo com estudo conduzido por Thieme (2017), os principais objetivos estratégicos da interação com startups para a grande empresa são adquirir conhecimento e atualização sobre novas tecnologias e modelos de negócios; desenvolver novas formas de fazer negócios, conquistando novos mercados e segmentos de clientes, e por fim, inovar em produtos, serviço e processos internos de forma mais ágil e mais barata. Para as startups, os benefícios da interação vão desde acesso ao conhecimento e infraestrutura de produção da grande empresa ao acesso ao mercado e canais de distribuição (Weiblen, Chesbrough, 2015).

As formas de interação são inúmeras e variam em função dos objetivos e grau de relacionamento buscado. A 100 Open Startups (2017), classifica os tipos de relacionamento entre grandes empresas e startup em quatro grupos principais: Relacionamento de Posicionamento; Relacionamento de Plataforma e Parcerias; Relacionamento de Desenvolvimento de Fornecedores e Relacionamento de Investimento.

Entre as interações de Relacionamento de Posicionamento estão as iniciativas de capacitação e mentoria, matchmaking e conexões, reconhecimento, premiações e espaços de coworking. As interações de Plataforma e Parcerias abrangem os vouchers de serviço e tecnologia, licenciamento de propriedade intelectual (PI) da grande empresa, acesso a recursos não-financeiros, acesso a base de colaboradores, acesso a base de clientes e canais de vendas. Os Relacionamentos de Desenvolvimento de Fornecedores envolvem recursos para P\&D e

R. Eletr. do Alto Vale do Itajaí - REAVI, v. 7, n. 11, p. 01-09, dez., 2018 ISSN: 2316-4190, DOI: $10.5965 / 2316419007112018001$ 
prototipagem, licenciamento de PI da startup, contratação de projeto piloto e fornecimento de serviço ou produto inovador. E por fim, os Programas de Relacionamento de Investimento compreendem programa de aceleração com equity, investimento com participação acionária minoritária e aquisição e incorporação. Estes grupos de relacionamento revelam o grau de intensidade e maturidade da relação que se estabelece com as startups (OPEN, 2017).

Em que pesem os benefícios da associação para uma startup, aliar-se a uma grande empresa também pressupõe pontos negativos que devem ser considerados. Weiblen and Chesbrough (2015) ressaltam que, se por um lado o dinheiro da grande empresa e a facilidade de acessar o mercado são fatores positivos para a startup, estar comprometido com um grande player pode limitar a sua liberdade de pivotar a ideia ou realizar negócios com concorrentes. Para os autores, uma eventual apropriação indevida da solução ou mesmo a demora na tomada de decisões pode colocar em risco a chance de sucesso da startup. Nesta mesma linha, Dushnitsky e Shaver (2009) discorrem sobre o risco da grande empresa querer defender unicamente seus próprios interesses, em detrimento da startup, movida pela vantagem competitiva que possui.

Podem também ocorrer conflitos no momento da valorização dos ativos das startups, impactando a geração de negócios entre as partes. É neste ponto que se abre outro campo de discussão interessante: o papel da propriedade intelectual nas relações de Corporate-Startup Engagement (CSE). Para uma startup, a propriedade intelectual é o seu principal ativo e na ausência de ativos tangíveis, os novos empreendimentos devem fornecer sinais confiáveis sobre a qualidade de suas inovações para potenciais investidores (Dushnitsky; Shaver, 2009).

Por um lado, a propriedade intelectual se coloca como um mecanismo para reduzir os custos de transação associados à estipulação de acordos entre a grande empresa e as startups, ou mesmo, um incremento na valorização da startup, aumentando seu poder de barganha. Por outro lado, não são todos os setores de atuação que se beneficiam de uma proteção via propriedade intelectual. Segundo Dushnitsky e Shaver (2009), algumas indústrias como as farmacêuticas são beneficiadas por fortes patentes, enquanto a indústria eletrônica, por exemplo, possui dificuldade em especificar e proteger circuitos eletrônicos, passíveis de engenharia reversa, tornando as patentes menos efetivas. Estas particularidades interferem na qualidade da interação entre as grandes empresas e startups.

De forma geral, startups que não possuem um sistema de proteção eficaz sobre a sua inovação se mostram menos propensas em abrir dados sobre o negócio, que por sua vez, são fundamentais para as grandes empresas prosseguirem com as negociações, pois revela-se importante o conhecimento da tecnologia desenvolvida e isso acaba diminuindo as oportunidades de realização negócio (Dushnitsky; Shaver, 2009). Os mesmos autores apontam que estas são preocupações reais que as startups devem ter porque, não raramente, acontece das grandes empresas estabelecerem um relacionamento para usufruírem indevidamente das inovações trazidas de fora, especialmente quando operam em um mesmo segmento de mercado, se baseiam em conhecimentos técnicos e possuem lógica de comercialização similares. Portanto, quando a startup opera em um regime de propriedade intelectual fraco, tende a abrir menos as peculiaridades do seu negócio, por medo de imitação, e isto impacta diretamente na sua valoração econômica quando das transações com a grande empresa (Dushnitsky; Shaver, 2009).

Outro desafio resultante desta interação, analisado por Hogenhuis, Hende e Hultink (2016), advém da divergência acerca da melhor etapa do processo inovativo para que a parceria possa render os melhores benefícios. Segundo os autores, para as grandes empresas as primeiras etapas do processo de inovação são mais interessantes, enquanto, para as startups, o valor está no final do processo, quando podem aproveitar dos canais de comercialização que as grandes empresas podem abrir. 
Embora diversos estudos tenham surgido nos últimos anos, a CSE é um movimento recente, cujos desafios ainda são pouco claros. Estudos conduzidos pela organização 100 OPEN STARTUPS em 2017, onde se analisam 613 contratos gerados entre startups e grandes empresas entre os anos de 2015 e 2017, evidenciam como as grandes empresas e startups ainda estão aprendendo a desenvolver negócios em conjunto. Das interações estudadas, $80 \%$ ainda são baseadas em processos que buscam aproximar os atores, mais do que efetivamente, estabelecer parcerias. Conforme ilustrado na figura 1, as premiações, a realização de eventos de conexão e mentoria e a disponibilização de espaços de coworking figuram como as iniciativas de maior destaque.

\section{Figura 1 - Tipos de Interações entre grandes empresas e startups.}

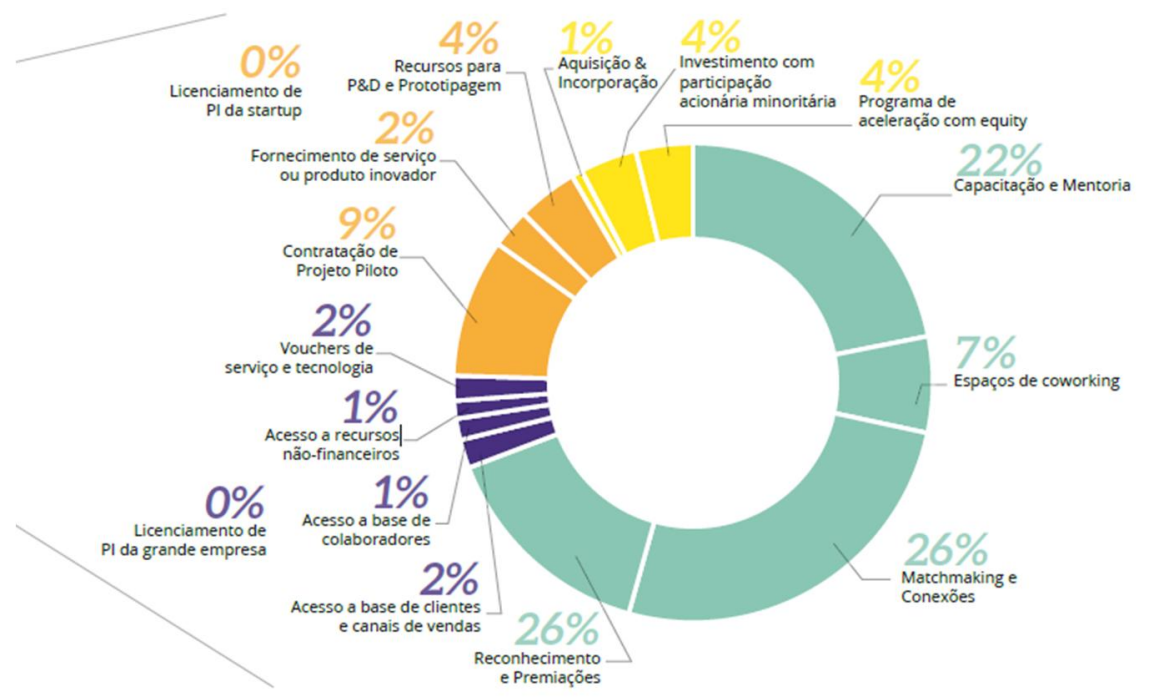

FONTE: 100 Open Startups, 2017

Embora estas interações contribuam para o desenvolvimento do ambiente de inovação, o fato de apenas $10 \%$ das interações se concretizarem em negócios para as empresas demonstra o baixo nível de maturidade do ecossistema no país. Chama atenção também que, no Brasil, nenhum contrato de licenciamento de propriedade intelectual tenha sido gerado entre as partes (Open, 2017). Estes dados sugerem que, embora o mercado esteja atento para as vantagens decorrentes dessas interações entre grandes empresas e startups, a maturidade das ações ainda é baixa. Este é um movimento recente, cujos erros e acertos ainda estão sob teste.

\section{Conclusão}

Pensar em sustentabilidade dos negócios a médio e longo prazos é pensar em inovação. Mais ainda, em inovação aberta.

A alta competitividade dos mercados, a celeridade dos avanços tecnológicos, bem como a massificação das tecnologias da informação e comunicação provocaram nas grandes empresas - tidas normalmente como tradicionais e conservadoras - uma nova estratégia de relacionamento com o ambiente externo: a parceria com empresas ágeis, descomplicadas e mais propensas a riscos - as startups.

Do ponto de vista das startups, a parceria com grandes empresas tem-se mostrado fundamental para o alcance da escala almejada para seus modelos de negócio, ampliando suas chances de sucesso.

Neste contexto, buscou-se analisar, sob o ótica das oportunidades e dos desafios, este tipo especial de inovação aberta que envolve a interação entre grandes empresas e startups.

R. Eletr. do Alto Vale do Itajaí - REAVI, v. 7, n. 11, p. 01-09, dez., 2018 ISSN: 2316-4190, DOI: $10.5965 / 2316419007112018001$ 
É fato que grandes empresas e startups possuem caraterísticas distintas porem complementares. Neste processo de interação, as empresas se beneficiam com o aumento de competitividade, soluções de problemas com menor custo e menor risco, agilidade e conhecimento tecnológico das startups. Por outro lado, as startups se beneficiam com o acesso a recursos financeiros, rol de clientes, canais de distribuição, infraestrutura e visibilidade, dentre outros aspectos proporcionados por quem tem nome e reconhecimento no mercado.

Entretanto, há que se considerar ainda os riscos inerentes a essas interações. O poder de mercado das grandes empresas pode, por vezes, significar na perda do poder de barganha das startups, assim como o regime de propriedade intelectual a qual estão submetidas as tecnologias envolvidas na negociação. Um erro na escolha do parceiro ou a associação em uma etapa equivocada do processo inovativo também são variáveis que podem comprometer o sucesso da parceria.

Apesar dos desafios, a necessidade de empresas e startups atuarem em conjunto já não é mais uma tendência e sim um fato. O crescimento das startups em todo o mundo demonstra uma nova forma de se fazer negócios, de reinventar modelos de negócios e de gerar inovação. A grande empresa está atenta a isto, buscando estabelecer vínculos e se aproximar destes atores.

Os processos de interação ainda estão sendo construídos e consolidados. As partes envolvidas precisam aprender a se relacionar, firmando parcerias sólidas e consistentes e diminuindo os riscos associados a estas interações, de forma a aumentar as vantagens para ambas.

\section{Referências}

100 Open Startups. Como grandes empresas e startups se relacionam. 2017. Disponível em: $<$ https://www.openstartups.net/br-pt/\#challenges>. Acesso em: 04 abr. 2018.

ABDI. Conexão Startup Indústria: Prestação de contas 2017. Brasília, 2017.

BERGER, C. et al. Co-designing modes of cooperation at the customer interface: Learning from exploratory research. European Management Review, 2005.

BLANK, S.; DORF, B. The Startup Owner's Manual: The Step-by-Step Guide for Building a Great Company. K\&S Ranch. Inc. Publisher, Pescadero, CA, 2012.

CHESBROUGH, H. Open Innovation: The New Imperative for Creating and Profiting from Technology. Harvard Business School Press, Boston, 2003.

CHESBROUGH, H.; VANHAVERBEKE, W.; WEST, J. Open Innovation: Researching a New Paradigm. Oxford University Press, Oxford, 2006.

CHIESA,V.; MANZINI, R.; PIZZURNO, E. The externalisation of R\&D activities and the growing market of product development services. R\&D Management, p. 65-75, 2004.

DOCHERTY, E. Primer of Open Innovation: Principles \& Practice, PDMA Visions, p. 13-17, 2006.

DUSHNITSKY, G.; SHAVER, J. M. Limitations to interorganizational knowledge acquisition: the paradox of corporate venture capital. Strategic Management Journal. 2009 
ENGEROFF, R.; BALESTRIN, A. Inovação fechada versus inovação aberta: um estudo de caso da indústria de cutelaria. In: Simpósio de Gestão da Inovação Tecnológica, Anpad, Brasília, 2005.

GIL, A. C. Como elaborar projetos de pesquisa. 4. ed. São Paulo: Atlas, 2002.

GRAHAM, P. Startup = growth. Disponível em: <http://paulgraham.com/growth.html $>$. Acesso em: 13 ago. 2018

HOGENHUIS, B.; HENDE, E.; HULTINK, E. J. When Should Large Firms Collaborate with Young Ventures? Research-technology Management, pages 39-47, 2016.

HUIZINGH, E. Open innovation: State of the art and future perspectives. Technovation, Groningen, pages .2-9, 2011.

LAZZAROTTI, V.; MANZINI, R. Different Modes of Open Innovation: A Theoretical Framework and an Empirical Study. International Journal of Innovation Management, 2009.

NIRAZAWA, A. Inovação nas Organizações. 2015.

OCDE. Francati Manual: Proposed Standard Practice for Surveys on Research and Experimental development. 2002.

RAHMAN, H., RAMOS, I. Open Innovation in SMEs: From Closed Boundaries to Networked Paradigm. Issues in Informing Science and Information Technology. v. 7, 2010.

SANTOS, A. R. DOS. Metodologia científica: a construção do conhecimento. 6. ed. rev. Rio de Janeiro: DP\&A, 2006.

STAL, E.; NOHARA, J.; CHAGAS JR., M. Os conceitos da inovação aberta e o desempenho de empresas brasileiras inovadoras. Revista de Administração e Inovação, pages 295-320, 2014.

SUDATI, E. Analysis of the Strategic Interaction among Established Corporations and Startups in Italy. Dissertação, Politecnico Milano 1863, Milão, Italia, 2016.

THIEME, K. The strategic use of corporate-startup engagement. Dissertação, Delft University of Technology, Holanda, 2017.

VARRICHIO, P. C. Uma discussão sobre a estratégia de inovação aberta em grandes empresas e os programas de relacionamento voltados para startups no Brasil. Racef - Revista de Administração, Contabilidade e Economia da Fundace, pages.148-161, Ribeirão Preto, 2016.

VERMEERSCH, I; VANHAVERBEKE, W; ZUTTER, S. Open innovation in SMEs: How can small companies and start-ups benefit from open innovation strategies? Research Report, Flanders, 2012. 


\section{11 Universidade do Estado de Santa Catarina Centro de Educação Superior do Alto Vale do Itajaí}

WEIBLEN, T; CHESBROUGH, H. Engaging with startups to enhance corporate innovation. California Management Review, pages 66-90, 2015. 